\title{
Mobilizing Undergraduate Students to Develop Practical Bioinformatics Databases and Analysis Tools for Biologists
}

\section{Qunfeng Dong ${ }^{1,2 *}$}

${ }^{1}$ Center for Biomedical Informatics, USA

${ }^{2}$ Department of Public Health Sciences, Stritch School of Medicine, Loyola University Chicago, 2160 S. First Avenue, Maywood, Illinois, USA

Despite the rapid advances in bioinformatics in recent decades, the development of computational software suitable for practical use still lags significantly behind the growing demand from biologists. Often this is due not to the technical challenges of software development, but rather to a serious shortage of developers available to write the software. Although the number of bioinformatics graduate students, postdoctoral fellows, and full-time staff has been increasing, this has not been enough to control the software shortage due to the complicated realities of these jobs. For instance, in the interest of career development, these individuals may feel the need to work on theoretically-minded research or large-scale projects rather than developing applied or smaller-scale utilities to solve some of the problems that biologists face day-to-day. As a result, development of practical bioinformatics software remains inadequate, and biologists are left frustrated that they lack the necessary computational tools to complete their work. This situation must be improved in order to meet the demand for practical bioinformatics software.

We believe it is important to recruit and train undergraduate students - a resource with a vast amount of under-utilized talent - to develop such practical software by involving them in our actual research projects. We have seen success with this strategy firsthand while working with undergraduates to develop Web-based bioinformatics software and databases; for the many biologists without specialized computational resources or technical training, such "cloud-based" services are invaluable enablers of their research, and in return our undergraduates have earned authorships in peer-reviewed research papers $[1,2]$. Undergraduate students bring to the table a significant amount of software development skill as well as enthusiasm for solving practical problems. Working on these research projects provides the opportunity for young students to make significant contributions while taking some of the burden off of graduate students, fellows, and staff. The experience will also prepare the students to become qualified researchers on advanced projects and allow them to show off complete software products of their own handiwork. Even better, bioinformatics projects can attract students from diverse disciplines, including biology, computer science, and engineering, and allow them to put their individual skills to use and to collaborate with one another. For example, students interested in programming could write the code, biology students could test the programs and provide input on usability and desirable features, and the two could work together to write documentation and tutorials. In this way, students from these different disciplines will be able to learn from one another. It is important to realize that undergraduate students have already acquired the basic skills needed to participate in these projects, e.g., genomics for biology students and programming for computer-science students. The projects will challenge and motivate the students to expand their knowledge and skill sets, providing a mutually beneficial arrangement between the bioinformatics labs and the students. Properly recruited and trained, undergraduate students can be an excellent way to address the everpresent need for practical software development in bioinformatics.

\section{References}

1. Revanna K, Munro D, Gao A, Chiu C, Pathak A, et al. (2012) A web-based multi-genome synteny viewer for customized data. BMC Bioinformatics 13: 190.

2. Revanna K, Chiu CC, Bierschank E, Dong Q (2011) GSV: A web-based genome synteny viewer for customized data. BMC Bioinformatics 12: 316 .
*Corresponding author: Qunfeng Dong, Department of Public Health Sciences, Stritch School of Medicine, Loyola University Chicago, 2160 S. First Avenue, Maywood, Illinois, USA, Tel: 708-327-9004; E-mail: qdong@luc.edu

Received October 23, 2015; Accepted October 27, 2015; Published October 30, 2015

Citation: Dong Q (2015) Mobilizing Undergraduate Students to Develop Practical Bioinformatics Databases and Analysis Tools for Biologists. Next Generat Sequenc \& Applic 2: 121. doi:10.4172/2469-9853.1000121

Copyright: (C) 2015 Dong Q. This is an open-access article distributed under the terms of the Creative Commons Attribution License, which permits unrestricted use, distribution, and reproduction in any medium, provided the original author and source are credited. 